\title{
Functional Neuroimaging Studies of Obesity: Linking Neuroscience to Health Behavior
}

\author{
Cary R. Savage, Director, Center for Health Behavior Neuroscience; John H. \\ Wineinger Professor of Psychiatry and Behavioral Science, University of \\ Kansas Medical Center
}

$\mathrm{T}$

The theme of this year's Merrill Retreat was "Behavioral and Social Sciences as Key Components in National Research Initiatives." I was invited to participate as a Research Faculty panel member. In this role, I presented a short overview of our research efforts aimed at understanding the neurobiological mechanisms underlying overeating and obesity and how we are moving these advances in neuroscience into the realm of clinical and behavioral intervention. I believe our efforts from the KUMC Center for Health Behavior Neuroscience provide good examples of the fundamental importance of behavioral and social sciences to advancing national research initiatives on the obesity epidemic. In this paper, I will overview the obesity problem and our research efforts aimed at defining brain function differences between obese and healthy weight groups, and then linking brain function to success or failure in diet and exercise interventions.

The "Epidemic" of Obesity. Obesity rates are on the rise and associated with serious public health consequences and rising health care costs.

Overweight and obesity are defined by a body mass index (BMI) of 25 to 29.9 and 30 or greater, respectively. A certain percentage of the human population has always been obese. Before 1990, approximately $10-14 \%$ of adults in the U.S. were obese as defined by a BMI $>30$. Since 1990, however, rates of obesity have increased dramatically, to the point that over $30 \%$ of the U.S. adult population is now obese (Ogden et al., 2006). In fact, if rates continue unabated, approximately $75 \%$ of the adult American population will be overweight or obese by the year 2020 (Wang et al., 2011). Increases in obesity prevalence in children are com- parable to those found in adults (Ritchie et al., 2003) and portend continued increases among adults.

Obesity is associated with significant public health consequences. Both overweight and obesity are characterized by the accumulation of excess levels of body fat and contribute to Type 2 diabetes, heart disease, hypertension, stroke, some cancers, osteoarthritis, as well as psychosocial and economic difficulties such as work disability (Mokdad et al., 2003). Not surprisingly, obesity also has dramatic impacts on increasing health care costs. Recent estimates of the total costs of obesity in the U.S. are over 140 billion dollars per year (Finkelstein et al., 2009). There are also secondary costs of obesity that may be surprising. For example, cars now burn more gaso- 
line to transport our larger body masses. In 2006, Jacobson and colleagues (Jacobson et al., 2006) estimated costs of increased automobile gasoline consumption attributable to obesity at 2.2 billion dollars per year. Using these estimates and current gasoline prices ( $\$ 3.50 /$ gallon), obesity increases automobile gasoline costs in the U.S. by approximately 3.5 billion dollars per year. This is cars only; other costs of transportation (e.g., airlines) are similarly increased.

Given the demographic changes, associations with negative health consequences, and economic burden, it is easy to justify the use of labels such as "epidemic" to describe the obesity problem in the U.S. and other developed countries. On a more optimistic front, weight loss has been shown to diminish risk for disease. Numerous studies have shown the beneficial effects of diminished weight and body fat in overweight and obese individuals. Weight loss has been shown to reduce blood pressure, improve cholesterol, improve glucose tolerance, and reduce markers of inflammation (associated with heart disease). While weight loss of $10 \%$ is generally recommended, improvements in disease risk factors have been demonstrated with as little as $2-3 \%$ weight loss (Truesdale et al., 2005). There are, therefore, strong reasons to develop better interventions for obesity.

Societal Changes Leading to the Obesity Epidemic. We live in a society where food is plentiful and exercise is optional.

Obesity is a complex medical and behavioral problem that boils down to a very simple cause: consistent energy in- take in excess of daily energy requirements. The old adage "calories in vs. calories out" is true, and changes at the societal level have contributed to chronic energy imbalances and, therefore, obesity.

Until very recently in our evolutionary history, acquisition of calories for consumption required work. First, we hunted over long distances for animals and plants. In fact, humans lived nomadic lifestyles, constantly on the move in search of sources of food and water. Later, farming and livestock management techniques allowed us to remain geographically stable, but even these methods required long hours of physical labor. This is no longer the case. Americans and other western citizens now live a privileged life. We drive from place to place in comfortable, climatecontrolled cars. We complain if we have to walk more than a few feet from our cars to our places of work. We spend our days in the sitting position, working on computers. We even use "drive-up windows" to acquire food. We can, without expending any calories beyond baseline metabolism, drive up to a window, and for a few dollars, have literally thousands of calories handed to us from a window.

Food portion sizes have also increased. An article in National Geographic Magazine (Newman, 2004) includes a startling graphic summarizing changes in portion sizes of common food and drink items. For instance, average portion sizes for a cheeseburger have increased from 202 calories to 310 calories; fries from 210 calories to 610 calories; a serving of "Coca-Cola" from 79 calories to 194 calories; a serving of pop- 
corn from 174 calories to 1,700 calories. Even our leisure is now effortless. The average American spends 2.7 hours per day watching television and another half-hour per day on a computer for leisure (Bureau of Labor Statistics, 2010).

Given all these changes in lifestyle, it is easy to see how energy imbalances occur and add up over the years to create obesity. Magnified across a population, we end up with a societal obesity epidemic. The solution is easy - eat less and exercise more - yet, it is highly elusive. Though the population tipping point has arisen from large-scale societal changes, biology plays a critical role in determining poor health choices at the individual level. Research is now aimed at understanding the biological roots of this resistance to healthy decisionmaking.

Eating and the Brain. Why it is so hard to "Eat Less and Exercise More"

I noted previously that the availability of plentiful, calorie dense, food is a recent development in modern society. It turns out that our brains have not kept up with these rapid changes. We remain biologically driven to consume calories whenever possible and move as little as needed to conserve energy. These drives are largely regulated by the brain. Health behaviors, such as eating, are influenced by a convergence of processes in the brain, including homeostatic factors and motivational and reward processing. Motivation and reward processing are especially important contributors to overeating and sedentary lifestyle in humans (Wang et al 2004). For example, food is a highly salient reinforcer (Epstein et al 2007) and its presentation is associated with increased activi- ty in limbic and paralimbic networks in the brain - these very brain regions play critical roles in helping us prioritize our attentional and behavioral resources. Abnormal activity in these networks may lead to increased susceptibility to overeating and other maladaptive health choices. As such, overeating and obesity may be conceptualized as reflecting failures in impulse control that are associated with unique patterns of brain activity.

Despite our biological programming, not all people become obese. Even dire predictions for the obesity epidemic predict that $25 \%$ of the U.S. population will remain healthy weight in 2020 (Wang et al., 2011). There are clearly important individual differences in the drive to eat and remain sedentary. The remainder of this chapter will focus on our attempts to understand differences in brain function that contribute to overeating and sedentary lifestyle.

Neuroimaging Efforts - Defining Brain Function in Obesity. There is now consistent evidence of altered brain function in obese groups

Recent advances in functional neuroimaging techniques have provided the opportunity to noninvasively study brain function differences between obese and healthy weight groups. The most widely used neuroimaging techniques include positron emission tomography (PET) and functional magnetic resonance imaging (fMRI). PET uses radioactively labeled isotopes to measure brain glucose metabolism or blood flow, while fMRI takes advantage of unique properties of oxygenated and deoxygenated hemoglobin in a magnet to provide measures of hemodynamic response (changes in brain blood flow and oxy- 
genation) associated with changes in mental state.

Although few in number, initial studies in obese adults have identified specific modifications of function in neural networks in comparison to healthy weight subjects. One research group produced most of the early published studies of obesity (Gautier et al 2000; 2001; DelParigi et al 2002; DelParigi et al 2005), all using PET and liquid meal tasting after prolonged fast. For example, DelParigi and colleagues (2002) used PET to examine regional cerebral blood flow in healthy weight and obese adults. Participants were scanned in two sessions, when hungry (after a 36 hour fast) and when consuming a satiating liquid meal. In the hungry condition, the obese group showed greater increases in brain blood flow to liquid tasting in the insula and cingulate cortex. After consuming the satiating liquid meal, differences were observed in the hippocampal formation, cingulate cortex, and amygdala. These findings implicate brain regions known to play a role in taste, reward, and behavioral control.

Our research team has now published several studies in obese and healthy weight groups. In these studies, we scan participants with fMRI as they view food and nonfood pictures (e.g., Bruce et al., 2010; Martin et al., 2010). We had previously validated these images in studies to identify food pictures that were maximally appetizing and nonfood pictures that were not appetizing but were nonetheless rated as equally positive and interesting. We scan participants in two sessions: Once after fasting at least four hours (Pre-meal condition) and once after eating a healthy $500 \mathrm{kcal}$ meal (Post-meal condition). This approach enables us to examine brain function differences during states of high food motivation (Pre-meal) and lower food motivation (Post-meal). This approach can be used in both adult (Martin et al., 2010) and pediatric (Bruce et al., 2010) populations. We have found that both obese children and adults show greater brain activation to appetizing food pictures in brain regions similar to those found in previous studies studying brain function while consuming liquid meals. We have also demonstrated that these differences in brain function are correlated with self-report ratings of eating behavior. For example, Figure 1 illustrates a result from Martin et al. (2010), highlighting a region in medial prefrontal cortex that is hyperresponsive in obese subjects and also

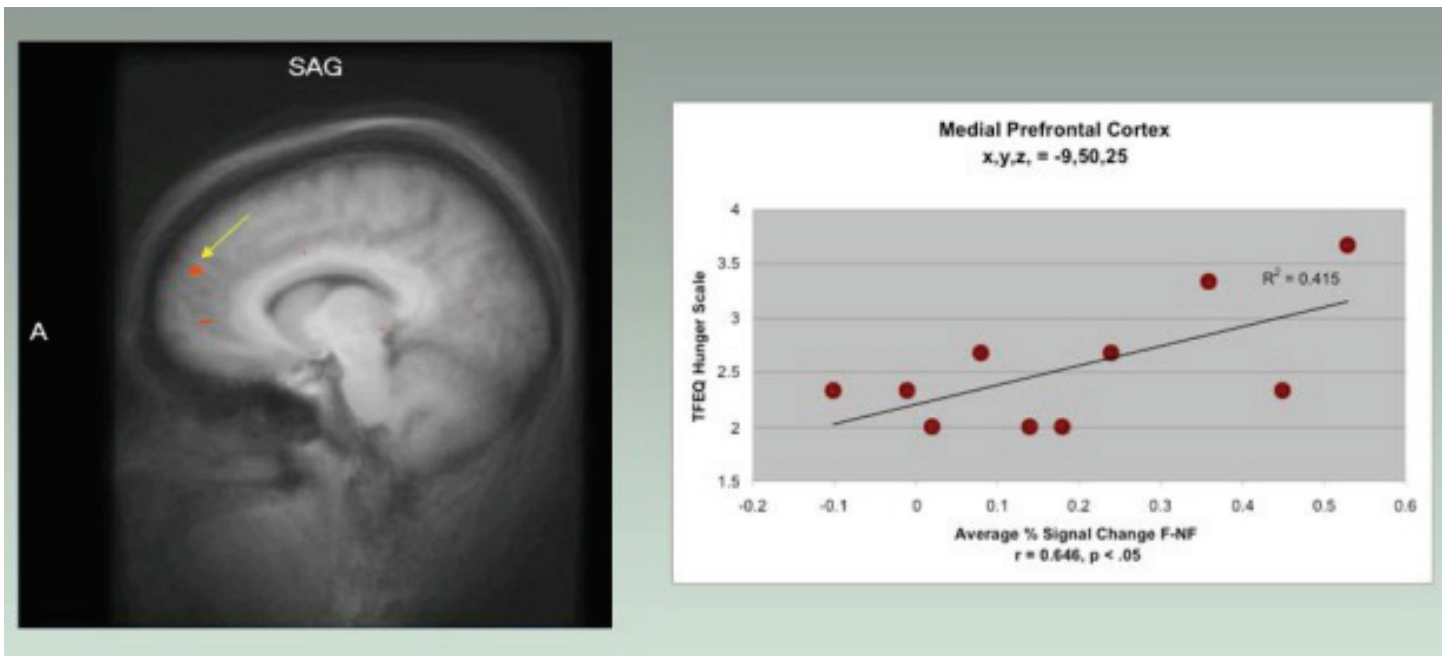

Figure 1 
positively correlated with self-report ratings of hunger.

Thus, our initial functional imaging studies have identified brain regions that respond differently to visual food cues in obese and healthy weight individuals, and are positively correlated with reports of hunger in obese participants. These brain areas are known to be involved in the processing of taste, reward, and behavioral/cognitive control. While shedding some light on mechanisms of overeating, many important questions remain. For instance, it is not yet known whether brain activation patterns change after dieting, or if they change differentially in successful and unsuccessful dieters. In addition, little is currently understood regarding biological processes that contribute to longterm maintenance of healthy weight. These missing pieces highlight the need to link neurobiological findings to actual health behaviors.

Linking Neuroscience to Health Behavior - Diet. We are investigating brain function predictors of weight loss and weight loss maintenance in diets

One major goal of our current research efforts is aimed at linking brain function differences to different outcomes in diet interventions. To achieve this goal, we established a collaboration with the Energy Balance Laboratory of Dr. Joseph Donnelly. We received NIH R01 funding in 2008 (DK080090) to support a project in which we scan obese and healthy weight participants with a the food motivation fMRI paradigm, described previously, during a baseline state and after obese participants have completed a twelve-week diet-based weight loss intervention. Obese partici- pants are then followed though a 6month weight maintenance period. Our project has three Specific Aims:

1. Characterize brain activation underlying increased food motivation and impulsive eating in obese individuals.

2. Identify brain activation predictors of initial weight loss.

3. Identify brain activation predictors of weight loss maintenance.

We have now completed analyses of fMRI data from the Baseline session. In these analyses, we examine fMRI predictors of future success or lack of success in the 3 month diet intervention. "Successful dieters" are defined as those who lose at least $7 \%$ of starting body weight, while "unsuccessful dieters" lose less than $7 \%$. Initial analyses indicate that the future unsuccessful dieters show two important differences in brain activity when viewing food pictures before dieting: First, they have decreased activity in prefrontal cortex; second, they show increased activity in the sensorimotor regions of the brain corresponding to mouth and tongue. Thus, our preliminary data indicate that unsuccessful dieting is predicted by decreased activity in parts of the brain implicated in behavioral inhibition and control (prefrontal cortex) and increased activity in the areas of the brain controlling the mouth and tongue. These results provide important clues about resistance to weight loss in diets. We are now analyzing the longitudinal data from the study in order to identify predictors of 6-month weight loss maintenance.

Linking Neuroscience to Health Behavior - Exercise. We are also investigating brain function predictors of adherence to 
exercise programs and the beneficial effects of exercise on brain function

Our group has more recently begun a collaboration with the Energy Balance Laboratory on an NIH R01 (DK085605) funded study designed to examine predictors of adherence to a 9-month exercise program. Regular exercise is a critical component of maintaining energy balance and it is associated with health benefits, including enhanced brain function. Health-related decisions, such as whether to exercise, are influenced by a convergence of processes in the brain, as individuals weigh the perceived balance between the rewarding and punishing aspects of the behavior, and whether gratification is immediate or delayed. Healthy behaviors are in part difficult to maintain because they are less immediately gratifying. In fact, exercise may initially be perceived as aversive. Despite this challenge, some people are able to persevere and experience long-term benefits. We hypothesize that brain processes underlying reward processing and impulse control will help us better understand mechanisms of obesity and health-related decision making. With this goal in mind, we are scanning participants as they gain and lose money and as they make monetary choices between small immediate rewards and larger delayed rewards. Scanning will take place before and after a nine-month exercise intervention.

The study addresses three Specific Aims:

1. Characterize brain activation underlying reward processing and impulse control in obese and healthy weight individuals.
2. Identify brain activation predictors, from the Baseline Session, of adherence and success in the exercise program.

3. Identify the beneficial effects of exercise and increased fitness on brain activation.

We predict that measures of brain activation will be useful in identifying neural contributions to obesity, determinants of adherence to a long-term exercise program, and the beneficial effects of exercise on brain function. This study is currently underway.

Summary. Early attempts to link behavioral and social sciences to obesity research initiatives show real promise

Obesity is a critical and growing problem in the U.S., with alarming health and economic ramifications. Previous work has linked obesity to altered functioning in brain networks supporting taste, reward, and cognitive control. The next challenge is to link these brain function differences to interventions designed to moderate food consumption and increase physical activity. The Center for Health Behavior Neuroscience was formed at the University of Kansas Medical Center (KUMC) in 2010 to support and unify research efforts at KUMC that are focused on brain function contributions to obesity, addiction, and other health behaviors. This paper has summarized some of our initial efforts aimed at linking neuroscience findings to healthy and unhealthy behavioral choices and response to intervention. As such, it highlights the importance of "Behavioral and Social Sciences as Key Components in National Research Initiatives." 


\section{$\underline{\text { References }}$}

Bruce AS, Holsen LM, Chambers R, Martin L, Brooks WM, Savage CR. Obese children show hyperactivation to food pictures in brain networks associated with motivation and reward. International Journal of Obesity 2010; 34:14941500.

Bureau of Labor Statistics. American Time Use Survey (2010). http://www.bls.gov/news.release/atus.nr0.htm

DelParigi A, Chen K, Salbe AD, Reiman EM, Tataranni PA. (2005). Sensory experience of food and obesity: A positron emission tomography study of the brain regions affected by tasting a liquid meal after a prolonged fast. NeuroImage 24:436-443.

DelParigi A, Gautier JF, Chen K, Salbe AD, Ravussin E, Reiman E, Tataranni PA. (2002). Neuroimaging and obesity: Mapping the brain responses to hunger and satiation in humans using positron emission tomography. Ann NY Acad Sci 967:389-397.

Epstein LH, Leddy JJ, Temple JL, Faith MS. (2007). Food reinforcement and eating: A multilevel analysis. Psychol Bull 133:884-906.

Finkelstein EA, Trogdon JG, Cohen JW, Dietz W. (2010). Annual medical spending attributable to obesity: Payer-and-service-specific estimates. Health Affairs 28:822-831.

Gautier JF, Chen K, Salbe AD, Bandy D, Pratley RE, Heiman M, Ravussin E, Reiman EM, Tataranni PA. (2000). Differential brain responses to satiation in obese and lean men. Diabetes 49:838-846.

Gautier JF, DelParigi A, Chen K, Salbe AD, Bandy D, Pratley RE, Ravussin E, Reiman EM, Tataranni PA. (2001). Effect of satiation on brain activity in obese and lean women. Obes Res 9(11):676-684.

Jacobson SH, McLay LA. (2006). The economic impact of obesity on automobile fuel consumption. The Engineering Economist 51:307323.

Martin LE, Holsen LM, Chambers RJ, Bruce AS, Brooks WM, Zarcone JR, Butler MG, Savage CR. Neural mechanisms associated with food motivation in obese and healthy weight adults. Obesity 2010; 18:254-260.

Mokdad AH, Ford ES, Bowman BA, Dietz WH, Vinicor F, Bales VS, Marks JS. (2003). Prevalence of obesity, diabetes, and obesity-related health risk factors, 2001. JAMA 289:76-79.

Newman C. (2004). The heavy cost of fat. National Geographic Magazine. August.

Ogden CL, Carroll MD, Curtin LR, McDowell MA, Tabak CJ, Flegal KM. (2006). Prevalence of overweight and obesity in the United States, 1999-2004. JAMA 295:1549-1555.

Ritchie LD, Ivey SL, Woodward-Lopez G, Crawford PB. (2003). Alarming trends in pediatric overweight in the United States. Soc Praventivmed 48:168-177.

Truesdale KP, Stevens J, Cai J. (2005). The effect of weight history on glucose and lipids: The Atherosclerosis Risk in Communities Study. Am J Epidemiol 161:1133-1143.

Wang GJ, Volkow ND, Telang F, Jayne M, Ma J, Rao M, Zhu W, Wong CT, Pappas NR, Geliebter A, Fowler JS. (2004). Exposure to appetitive food stimuli markedly activates the human brain. NeuroImage 21:1790-1797.

Wang YC, McPherson K, Marsh T, Gortmaker SL, Brown M. (2011). Health and economic burden of the projected obesity trends in the USA and the UK. Lancet 378:815-825. 